\title{
Lidil
}

Revue de linguistique et de didactique des langues

$51 \mid 2015$

Acquisition du langage et phonologie

\section{Comment comparer les éléments syllabiques et articulatoires des proto-mots et des mots au regard de la théorie de l'optimalité et de la théorie du Cadre/Contenu?}

How to Compare the Syllabic and Articulatory Elements of Protowords and Words Using the Optimality Theory and the Frame/Content Theory?

\section{Guillaume Roux}

\section{OpenEdition \\ Journals}

Édition électronique

URL : http://journals.openedition.org/lidil/3754

DOI : 10.4000/lidil.3754

ISSN : 1960-6052

Éditeur

UGA Éditions/Université Grenoble Alpes

\section{Édition imprimée}

Date de publication : 30 mai 2015

Pagination : 169-197

ISBN : 978-2-84310-297-4

ISSN : $1146-6480$

\section{Référence électronique}

Guillaume Roux, « Comment comparer les éléments syllabiques et articulatoires des proto-mots et des mots au regard de la théorie de l'optimalité et de la théorie du Cadre/Contenu ? », Lidil [En ligne], 51 | 2015, mis en ligne le 30 novembre 2016, consulté le 30 avril 2019. URL : http:// journals.openedition.org/lidil/3754; DOI : 10.4000/lidil.3754 


\title{
Comment comparer les éléments syllabiques et articulatoires des proto-mots et des mots au regard de la théorie de l'optimalité et de la théorie du Cadre/Contenu?
}

\author{
Guillaume Roux*
}

\begin{abstract}
RÉSUMÉ
Dans cet article, nous proposons d'explorer les relations entre notre méthode de travail et notre choix théorique concernant la comparaison des structures syllabiques et des paramètres articulatoires des protomots et des mots en français langue maternelle à partir d'un corpus longitudinal de 4 enfants français âgés entre 1 an et 2 ans. Notre objectif sera alors de justifier notre choix d'un corpus longitudinal pour explorer la problématique des proto-mots et des mots, ainsi que ceux de la théorie de l'optimalité pour la dimension syllabique et de la théorie du Cadre/ Contenu pour la dimension articulatoire.
\end{abstract}

\begin{abstract}
In this article, we propose to explore the relationships between our methodology and our theoretical choices concerning the comparison of syllabic structures and articulatory elements of protowords and words in french, using a longitudinal body of work of 4 monolingual french children between 1 and 2 years old. Our purpose is to justify our choice of a longitudinal body of work to explore the problem of protowords and words, and why, to confirm our methodology, we chose the Optimality Theory for the syllabic dimension and the Frame/Content Theory for the articulatory dimension.
\end{abstract}

* Université de Provence, Laboratoire Parole et Langage (LPL), UMR 6057CNRS. 


\section{Introduction}

Dans cet article, nous nous proposons de discuter des choix théoriques et méthodologiques d'une recherche que nous avons menée sur la comparaison des proto-mots et des mots au niveau phonologique chez quatre enfants français monolingues âgés de un à deux ans. En effet, la période des premiers mots connait la présence simultanée de productions pouvant faire l'objet de catégorisations différentes (mots, non-mots porteurs de sens, babillage). Cette présence simultanée permet d'observer et d'étudier, sur une même période, les différences qui déterminent ces diverses catégories de productions de l'enfant, notamment au niveau phonologique, seul point commun de ces productions. Cette particularité unique dans le développement langagier de l'enfant permet alors d'observer, dans l'analyse de l'évolution de ces catégories, ce qu'il en est vraiment des enjeux phonologiques de cette période et de faire émerger les raisons profondes de ces différences. Ces raisons pourront être explicitée par le recours à deux perspectives théoriques qui peuvent sembler opposées, la théorie de l'optimalité ${ }^{1}$ pour le développement des structures syllabiques (Prince \& Smolinski, 1993) et la Frame/Content Theory (MacNeilage \& Davis, 1990) pour le développement articulatoire. En effet, l'OT permet de déterminer les raisons du passage rapide d'une période ayant tendance à la simplification syllabique (proto-mots) à une période manifestant une complexité syllabique plus grande et relevant d'un développement syllabique particulier (cf. partie 3.1) reposant sur la présence de contraintes phonologiques profondes; ces contraintes peuvent s'observer par les proto-mots qui persistent à un moment où les mots sont les productions les plus fréquentes : l'enfant revient, avec ces proto-mots ([igo] [mapa] [tigo], cf. Roux, 2014), aux structures de base constituées de syllabes simples sur le modèle VCV ou CVCV. La Frame/Content Theory, quant à elle, permettra d'expliquer le passage rapide d'une période où les sons articulatoirement complexes sont absents à une période où ils sont présents, et donc de montrer la relation avec la maturation articulatoire de l'enfant. Le développement et la stabilisation des phénomène syllabiques et articulatoires, exprimant le passage des proto-mots comme productions majoritaires aux mots comme productions majoritaires, permet de montrer la corrélation qui existe entre ces dimensions et le développement lexical de l'enfant, notamment avec l'explosion lexicale (Bloom, 1973; Kern et Gayraud,

1. Désormais OT. 
2007), notion controversée que nous retrouvons toutefois dans nos résultats. Il ne s'agira donc pas de les opposer mais de montrer que, les dimensions phonologiques et articulatoires existant dans le développement de l'enfant, chacune est la plus appropriée pour expliquer les phénomènes en jeu dans chaque dimension étudiée, et qu'une complémentarité, dans le sens d'une influence d'une dimension sur l'autre ou d'une influence réciproque, est envisageable. Nous expliquerons que la justification de cette perspective n'est pas un choix préalable à notre étude mais qu'elle s'est imposée par la particularité de nos résultats.

Dans un premier temps nous ferons le point sur les différentes définitions qui ont été données du proto-mot et du mot dans la littérature. Notre recherche nous a ensuite orienté sur le besoin de considérer les définitions et catégorisations du mot et du proto-mot. Nous nous sommes retrouvé dans la nécessité de valider ces définitions, multiples, variées et parfois contradictoires par un test de perception mené auprès de dix adultes, afin d'établir empiriquement la validité d'une ou de certaines d'entre elles. Une fois les définitions validées, nous avons opté pour un corpus longitudinal afin de mieux assurer le suivi et l'évolution des productions que nous souhaitons comparer. Nous avons observé les structures syllabiques simples et complexes et les structures articulatoires qui composaient les mots et les proto-mots chez les enfants témoins de notre échantillon.

\section{Méthode}

\subsection{Présentation des données}

Au niveau méthodologique, nous avons opté pour un suivi longitudinal d'enfants français monolingues afin de mieux pouvoir mettre en évidence l'évolution progressive de ces types de productions. En effet, opter pour une approche longitudinale permettait de mieux observer la progression de la fréquence de ces deux types de productions. Les productions de mots et de proto-mots ont été relevées en contexte dialogal, que l'énoncé soit initié par l'enfant ou par la mère. Notre échantillon a été constitué à partir d'un corpus de quatre enfants, Marie, du corpus NIMH de Lyon ${ }^{2}$, Madeleine et Théophile du Paris corpus ${ }^{3}$, sélectionnés

2. Corpus NIMH de Lyon, sous la direction de Catherine Demuth et Harriet Jisa.

3. Projet ANR CoLaJE, 2009-2012, <http://colaje.risc.cnrs>, responsable scientifique : A. Morgenstern. Morgenstern (2009); Morgenstern \& Parisse (2012). 
à partir de la base de données CHILDES, et Elouan, enfant que nous avons filmé de 4 mois à 4 ans (Roux, 2012). Dans notre corpus, nous avons donc deux filles, Marie et Madeleine, et deux garçons, Théophile et Elouan. Ils ont été filmés entre l'âge de un an et trois ou quatre ans selon les enfants et nous avons retenu les enregistrements entre un et deux ans pour les besoins de notre travail. Les enregistrements avaient lieu une à deux fois par mois selon les projets de recherche ( 2 fois pour le projet NIMH et 1 fois pour le projet CoLaJE et pour notre travail sur Elouan) durant une heure, en situation naturelle (à la maison le plus souvent, avec au moins un adulte, parent, grand-parent, nourrice, le plus souvent la mère, dans des activités ordinaires, bain, repas, jeux, etc.). Le nombre total d'heures enregistrées concernant notre propre travail est de 52 heures.

Le choix de suivre l'action en contexte naturel nous a permis d'observer des situations qui constituent, d'après Arborio et Fournier (1999) :

[...] une façon d'échapper au sentiment de dépossession face aux outils toujours plus sophistiqués de traitement des données, perçus comme des «boîtes noires», et aux interminables interrogations sur la construction sociale des catégories utilisées dans le dénombrement pour contrôler l'intelligibilité des données quantifiées. (p. 6)

Par cette méthode, nous avons souhaité faire émerger les particularités des proto-mots et des mots "[...] par la décomposition d'événements singuliers, par le repérage d'enchaînements d'actions » (ibid., p. 45). Nous nous sommes également basé sur des transcriptions au format CHAT (Codes for the Human Analysis of Transcripts) à partir du logiciel CLAN afin d'avoir un outil permettant la décomposition de ces «évènements singuliers ». Ainsi, le choix de l'observation longitudinale de l'évolution de la proportion de mots et de proto-mots nous a conduit à considérer la présence d'une sorte de phase de substitution (exemple 1), une inversion rapide des courbes, les proto-mots étant majoritaires avant $1 ; 04,>80 \%$ et les mots le devenant largement après $1 ; 07,>80 \%$; cette phase reste à expliquer en termes de développement phonologique (Roux, 2012), pour lequel l'approche longitudinale semble la plus adaptée (exemple 1).

\begin{tabular}{|l|l|l|}
\hline & Début/fin & Avant/après l'inversion \\
\hline Marie & $\mathrm{p}<0,05(1 ; 00.03$ et $2 ; 00.09)$ & $\mathrm{p}<0,05(1 ; 02.28$ et $1 ; 06.23)$ \\
\hline Madeleine & $\mathrm{p}<0,05(1 ; 00.05$ et $2 ; 02.06)$ & $\mathrm{p}<0,05(1 ; 03.18$ et $1 ; 07.15)$ \\
\hline Théophile & $\mathrm{p}<0,05(1 ; 00.09$ et $2 ; 00.20)$ & $\mathrm{p}<0,05(1 ; 04.01$ et $1 ; 10.00)$ \\
\hline Elouan & $\mathrm{p}<0,05(1 ; 00.06$ et $2 ; 01.20)$ & $\mathrm{p}<0,05(1 ; 04.22$ et $1 ; 10.21)$ \\
\hline
\end{tabular}

Exemple 1. - Évolution des proportions de mots (rouge) et de protomots (bleu) de Madeleine entre un an $(1 ; 00)$ et deux ans $(2 ; 00)$. 
Nous pouvons donc observer, dans la période des premiers mots, un palier basé sur le passage d'une présence majoritaire de proto-mots à une présence majoritaire de mots. Ce sont les raisons de ce passage qui nous conduiront à discuter de nos choix théoriques pour les expliquer. Nous nous baserons sur l'observation des dimensions syllabiques et articulatoires, sachant que ces dimensions et la stabilisation de ces paramètres sont corrélées à l'explosion lexicale observée dans nos données. La période des premiers mots, selon nous, pourrait donc se faire en deux temps, l'un avec l'apparition des mots, l'autre avec l'explosion lexicale, la transition s'effectuant par un phénomène de maturation phonologique et/ou articulatoire que nous expliquons par l'OT et la Frame/Content Theory.

Les définitions du mot et du proto-mot (cf. partie 2.2.1) s'opposent essentiellement au niveau de la fluctuation phonétique ou de la réitération phonologique. Ainsi, au niveau phonologique, qu'est-ce qui permet d'expliquer ce passage rapide des proto-mots aux mots? Nous avons analysé 3000 de ces productions avec le logiciel Praat (50 productions choisies par transcription et par enfant), qui nous a permis de détailler les productions de mots et de proto-mots via un découpage syllabique et phonétique (exemple 2).

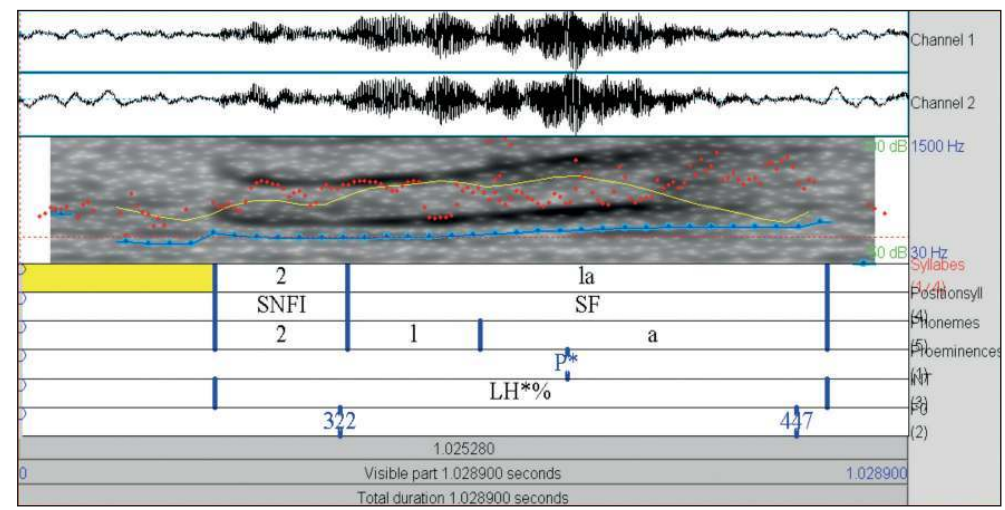

Exemple 2 . - Exemple de découpage d'une production sous Praat.

À partir de ces découpages, nous avons listé, pour les mots et les proto-mots, le nombre de structures syllabiques en fonction du type de structure rencontré (simple, complexe; V, CV, branchantes, codas). Considérant le phénomène de substitution rapide dévoilé par le recours à une approche longitudinale, ce point nous a orienté vers l'OT. En effet, nous cherchons à expliciter pourquoi la transition est rapide, notamment, 
au niveau sonore, au point d'exclure une dimension de confrontation à l'usage et de reconstruction. Nous avons effectué le même type de classification au niveau phonétique, selon la présence de caractéristiques articulatoires (occlusion, nasalité, etc.), ce qui nous a orienté vers la théorie du Cadre/Contenu. Ainsi, notre perspective se base, au regard des données mises en avant, sur une dynamique de maturation cognitive et articulatoire fondée sur des bases innées. Enfin, nous avons effectué des calculs de proportions et testé les différences, significatives ou non entre les proto-mots et les mots, et d'un âge à l'autre (test de $\mathrm{X}^{2}$ ).

Concernant l'émergence des phonèmes, dans la mesure où beaucoup de travaux ont analysé les relations entre le babillage et les mots au niveau vocalique et consonantique (Oller, 2000; Menn \& StoelGammon, 2005 ; Stoel-Gammon, 1985), et tout particulièrement dans le cadre de la Frame/Content Theory (MacNeilage \& Davis, 1990, 2000 ; Davis \& MacNeilage, 1995 ; Kern \& Davis, 2009; Davis \& Kern, 2011), nous n'avons pas pris le parti de nous baser sur le calcul du rapport C/V utilisé dans la Frame/Content Theory pour établir les relations babillage/mots et la prédictibilité articulatoire; en effet, notre travail se situe dans une perspective de comparaison entre les proto-mots et les mots. Nous avons alors calculé la variété vocalique (nombre de voyelles différentes par échantillon analysé sous Praat) dans les mots et dans les proto-mots à chaque âge et pour les quatre enfants. Nous avons calculé ensuite la diversité vocalique sur le rapport nombre de voyelles différentes/nombre total de mots ou de proto-mots. Nous avons effectué les mêmes calculs pour les consonnes.

Également, une fois nos unités définies, nous les avons relevées et nous avons réalisé un test inter-juges sur un échantillon de ces productions afin d'établir la qualité de notre jugement à l'aide d'un second codeur. S'il n'était pas possible de faire recoder tous les énoncés que nous avons analysés sur l'intégralité de notre corpus (trop de temps pour le second codeur), nous avons donc demandé à un second codeur linguiste de juger 10 mots et 10 proto-mots par enfant et par âge. Nous lui avons demandé de dire s'il s'agissait d'un mot ou non et de repérer la présence d'un sens identifiable. L'accord initial était de $95 \%$, puis une discussion sur les énoncés présentant un désaccord a permis d'établir un accord à $100 \%$. Il n'y eut donc pas de différence significative $\left(\mathrm{X}^{2}=7.4682\right.$, df $\left.=4, \mathrm{p}>0.05\right)$ entre notre identification et celle du second codeur (Roux, 2012).

Nous les avons comparées en termes de fréquence : il s'est avéré que les proto-mots sont des productions majoritaires au début de la deu- 
xième année, puis qu'un renversement rapide des proportions se produit dans le courant de la deuxième année, et qu'à la fin, les mots sont les productions majoritaires. Nous pouvons considérer que, pour chaque enfant, la répartition des pourcentages entre la première transcription et la dernière est radicalement et significativement différente. En revanche, la période de transition nous montre qu'en effectuant un classement par ordre de grandeur ( $\mathrm{X}^{2}$ de Mac Nemar) entre le début et la fin de la forte inversion des courbes, on observe qu'il n'y a pas de différence significative. Le lien entre les proto-mots et les mots semble alors établi. Ceci suggèrerait qu'il existe une sorte de phénomène de substitution des mots aux proto-mots comme modèle privilégié d'expression et de communication, et que cette substitution s'effectue dans une répartition similaire des proportions : il n'y a pas de différence significative dans les classements par ordre de grandeur entre le moment où ce sont les proto-mots et le moment où les courbes se sont inversées (exemple 3 ).

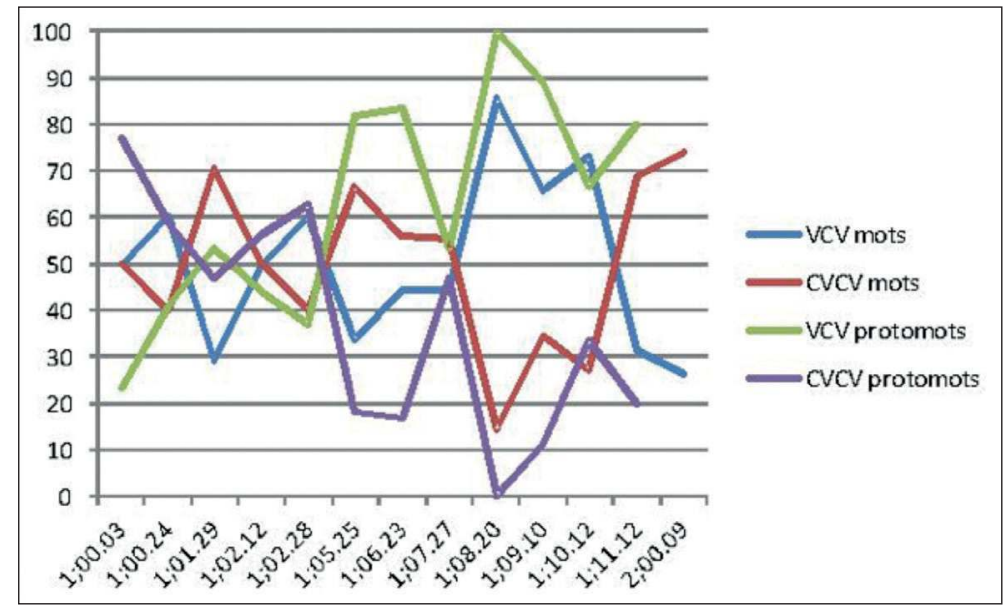

Exemple 3. - Rapport statistique des comparaisons de la distribution des proto-mots et des mots entre la première et la dernière transcription, et entre le moment précédent le phénomène de substitution et le moment succédant au phénomène de substitution chez Madeleine (test de $\mathrm{X}^{2} \mathrm{~d}^{\prime}$ indépendance et de Mac Nemar).

Dans la mesure où le test sur les adultes a permis de mettre en évidence que la différence majoritairement avancée concerne l'aspect articulatoire et sonore, il devient nécessaire, pour mieux identifier les causes des renversements de courbes, de comparer les structures syllabiques 
des proto-mots et des mots, afin de voir quels phénomènes sont présents ou non. C'est alors que la question du cadre théorique se pose. Mais nous avons jugé pertinent le fait que, dans notre travail, elle se pose à la fin. En effet, la définition du proto-mot et du mot n'a pas amené à proprement parler ce débat. Les différences que nous avons mises en évidence peuvent s'analyser et s'expliquer à partir de cadres théoriques divers, et même opposés comme la théorie basée sur l'usage (Tomasello, 2003) ou le minimalisme (Chomsky, 2005).

Quels choix théoriques et méthodologiques s'imposent pour la comparaison des structures syllabiques et articulatoires des proto-mots et des mots chez quatre enfants français monolingues entre un an $(1 ; 00)$ et deux ans (2;00)? La question qui pourrait se poser est de savoir, en fonction du constat de ces différences, quel cadre théorique est le plus à même d'expliquer le phénomène ou d'être accrédité par celui-ci.

\subsection{Définition et catégorisation des proto-mots et des mots}

\subsubsection{Définition des proto-mots et des mots}

Depuis les travaux de Menn (1978) dans lesquels figure la première occurrence de «protoword» (p. 24), tenant compte des travaux de Halliday (1975) sur le proto-langage, il est nécessaire de considérer les différentes approches antérieures afin d'établir précisément ce que sont ces deux catégories, et que les définitions données ne sont pas sans rapport avec un positionnement théorique et l'usage d'une méthodologie. Dans un travail précédent, nous avons établi un état de la question (Roux, 2014). Il s'avère que diverses définitions et dénominations ont été données pour ces productions ne recouvrant, selon les différents auteurs, pas les mêmes domaines : un mot inventé par l'enfant, une production idiosyncratique, une production imitée doivent-elles être catégorisées comme mot ou proto-mot?

Des particularités telles que l'importance de l'interprétation de la mère ont été mises en avant (Veneziano, 1981), ou ont été nuancées comme n'étant pas de premier plan pour identifier un mot (Vihman \& McCune, 1994). Suivant leur modèle des Vocal Motor Schemes (VMS), et considérant la production d'une ou plusieurs consonnes supra-glottales et récurrentes sur une période de temps donnée, le travail de ces dernières a permis d'établir qu'un mot est une forme phonologique réitérée dans une même situation et/ou désignant un même objet (incluant également les productions désignant plusieurs situations et plusieurs objets). Nous nous sommes beaucoup appuyé sur le travail de Vihman 
et McCune (1994), qui a conduit à valider ou non les critères d'identification d'une unité comme mot; néanmoins, leur travail ne concernant que l'identification des mots, nous avons considéré la nécessité de compléter ce qui concerne l'identification des proto-mots et ce qui les distingue des mots. Mais c'est à partir de leur travail, principalement, que nous avons pu envisager qu'un proto-mot serait une production phonétiquement fluctuante mais distinguable du babillage varié par la présence d'une intention de communication et d'une fonction sémiotique. Cependant, les définitions d'un chercheur à l'autre demeurent variables quant à établir la frontière stricte et précise entre un proto-mot et un mot, Vihman et McCune (1994) ayant, par exemple, nuancé les considérations de Veneziano (1981) sur l'identification du mot.

\subsubsection{Catégorisation des mots et des proto-mots}

Ainsi, dans un travail antérieur, nous nous sommes interrogé sur la fiabilité des catégorisations des chercheurs (Roux, 2014). Pour pallier cette limite, nous avons pris le parti de nous recentrer sur des catégorisations intuitives réalisées par des adultes. Nous avons eu recours à l'élaboration d'un test de perception (Roux, 2012, 2014). Nous avons confirmé ces définitions par ce test auprès d'adultes non familiers des domaines scientifiques concernés et des enfants de notre corpus, en leur faisant entendre des proto-mots et des mots préalablement sélectionnés selon les critères établis par les chercheurs (Menn, 1976; Vihman \& McCune, 1994; McCune \& Vihman, 2001), en leur montrant les contextes de production, mais sans définir d'ordre ni donner d'indice sur la nature de la production, et en les interrogeant sur la présence de sens et l'intelligibilité des productions. Les proto-mots et les mots ont été sélectionnés lorsque les 4 enfants en sont aux mêmes niveaux de MLU ${ }^{4}$. Le découpage en morphèmes permet, d'après Brown (1973), et Le Normand (1997) pour le français, d'observer le développement morphosyntaxique de l'enfant et de témoigner, par l'observation de l'unité minimale de sens qu'est le morphème, de l'existence de stades de développement, 10 mots et 10 proto-mots par enfant entendus par 10 adultes (exemple 4).

4. Les productions ont été sélectionnées lorsque les enfants ont tous, selon le critère du MLU basé sur le nombre de morphèmes moyen par énoncé (Brown, 1973; Le Normand, 1997), un stade de développement linguistique identique et sont au palier $\mathrm{I}$, avec une moyenne comprise entre 1,2 et 1,5 morphèmes par énoncé (Roux, 2012). 


\begin{tabular}{|c|c|c|c|c|c|c|c|c|}
\hline \multirow[b]{2}{*}{ Production } & \multicolumn{2}{|l|}{ Elouan } & \multicolumn{2}{|l|}{ Marie } & \multicolumn{2}{|l|}{ Madeleine } & \multicolumn{2}{|l|}{ Théophile } \\
\hline & Proto-mot & Mot & Proto-mot & Mot & Proto-mot & Mot & Proto-mot & Mot \\
\hline 1 & jaja & asi & evato & balõ & eja & $a R b R$ & œgi & bebe \\
\hline 2 & ydo & bal & tuve & banan & ada & asi & olo & kle \\
\hline 3 & lega & banan & putapa & bibR ว & meka & balõ & œра & fur $\int \varepsilon t$ \\
\hline 4 & batatø & bato & ake & bizu & depa & banan & adœ & Rã $3 \mathrm{e}$ \\
\hline 5 & lisa & bibR $\tilde{J}$ & gapa & blø & dad $\varepsilon$ & kado & nabœ & pik \\
\hline 6 & amo & bizu & mata & bet & bebø & $\int \mathrm{a}$ & epaja & fini \\
\hline 7 & $\mathrm{~d} \varepsilon \mathrm{d} \varepsilon$ & bobo & etitø & foset & uga & kofว̃ & tiga & $\mathrm{m} \tilde{\varepsilon}$ \\
\hline 8 & sase & bebe & lølø & Səval & mana & kulœR & memo & mamã \\
\hline 9 & yma & $\int \mathrm{a}$ & øve & flœR & igo & dodo & gigi & mãze \\
\hline 10 & ula & kase & køto & live & kapa & dwa & ejœ & poti \\
\hline
\end{tabular}

Exemple 4. - Liste des proto-mots (transcription phonétique) et des mots (transcription phonétique) sélectionnés pour le test.

Ce test a permis de montrer que les adultes identifient la présence de sens dans les deux types de productions; les proto-mots et les mots se caractérisent par la présence d'une fonction sémiotique. La différence entre les proto-mots et les mots repose sur la qualité de l'articulation de l'enfant, selon les adultes testés, plus fluctuante et moins précise en ce qui concerne les proto-mots.

À partir de ces résultats et des définitions antérieures (Vihman \& McCune, 1994 notamment), nous considérons donc comme mots :

- les unités ayant leur forme phonétique réitérée dans les mêmes situations et/ou pour désigner les mêmes objets indépendamment de leur proximité phonologique avec un mot de la langue. Nous incluons alors les productions inventées par l'enfant et réitérées ;

- les productions reconnues et interprétées par la mère ou un proche, phonologiquement proches de l'input et avec leurs versions allomorphiques, produites avec un contour intonatif marquant la présence d'une modalité de phrase, et réitérées par l'enfant dans des contextes similaires ou/et avec un/des sens identique(s);

- les productions imitées de l'adulte;

- les productions reprises par l'adulte si l'enfant les produit à plusieurs reprises;

- les productions avec généralisation et/ou surgénéralisation sémantique, comme les productions s'appliquant à toute une caté- 
gorie («papa» pour désigner tous les hommes), ou recouvrant de nombreux sens différents (mots-valises comme «lait» pour biberon, le lait, un contenant, un liquide quelconque);

- les onomatopées (par exemple «tchou tchou», «vroum vroum»);

- nous considérons les productions avec filler, lorsque le filler (phonème prélexical ou préverbal destiné à devenir un déterminant, un pronom ou un auxiliaire) est identifié comme étant au stade pré-morphologique ou proto-morphologique, comme un seul bloc ([eklé] pour «la clé» ou «une clé» ou «des clés»ou encore «les clés $)$;

- nous comptons les mots lexicaux, les déictiques «là» ou «ça».

Grâce aux éléments apportés par notre test de perception, nous avons catégorisé comme proto-mots :

- les productions phonétiquement fluctuantes (Menn, 1978) proches du babillage varié sur le plan phonologique (phonèmes alternés, structures syllabiques simples comme «mapo»), mais avec une intention de communication et un sens;

- les productions non interprétables, mais accompagnées d'un pointage;

- toutes les productions ne répondant ni à la définition de mots ni à de simples productions de voix (vocoïdes simples, cris, jasis, vagissements), ni à du babillage varié (avec des productions comme «ite» pour ballon, «ado» pour voiture);

- ainsi que les productions n'entrant pas dans la catégorie mots mais avec une modalité de phrase de la langue («mapo» avec un contour intonatif déclaratif ou interrogatif, simple montant ou simple descendant).

Pour être plus précis avec nos identifications, nous avons exclu les productions pour lesquelles nous avions des doutes, les productions trop chevauchées par un bruit, les productions chantées, les productions murmurées ou soufflées (sans voisement), les productions à l'intérieur de cris (cris articulés) ou de pleurs ou de chouinements ou de grognements. Nous avons exclu toutes les productions relevant du babillage varié n'ayant pas intuitivement un sens ou relevant d'une intention de communication, toutes les vocalisations ne répondant pas aux critères de définition des proto-mots ou des mots. 


\section{Résultats}

\subsection{Définition du mot et du proto-mot dans la littérature}

Dans nos résultats, nous constatons le fait que les structures syllabiques complexes (de type CVC ou CCV ou encore CCVC, CVCC et CCVCC) sont absentes des proto-mots chez les quatre enfants. Nous notons que cette absence est caractéristique de structures syllabiques qui n'ont pas la même difficulté articulatoire, les CVC étant plus difficiles à réaliser que les CCV (Demuth \& Johnson, 2003; Kern \& Gayraud, 2007; dos Santos, 2007); néanmoins, nous ajoutons que si elles sont plus difficiles à réaliser, les $\mathrm{CCV}$, plus simples, sont également absentes de nos résultats pour les proto-mots. Nous tenons cependant à souligner que cette particularité n'est relative qu'à nos données analysées, et que nous ne saurions être amené à en faire une vérité générale. Ce qui semble apparaitre toutefois est une fréquence d'apparition de ces structures moindre dans les productions de proto-mots que dans les productions de mots.

Dès lors, deux autres aspects intéressants ont pu être observés :

- le premier aspect concerne la répartition des proportions des structures simples commençant par un noyau, de type VCV ou VCVCV (que nous avons noté $\mathrm{VCV}(\mathrm{CV})$ ) par rapport aux structures commençant par une attaque, de type $\mathrm{CVCV}$ ou $\mathrm{CVCVCV}$ (que nous avons noté $\mathrm{CVCV}(\mathrm{CV})$ ) pour les productions de mots et de proto-mots;

- le second aspect concerne l'évolution de la fréquence d'apparition des structures complexes avec coda (CVC) et avec attaque branchante (CCV) (exemple 5). La question est de savoir s'il y a des différences, au niveau de ces structures, entre les proto-mots et les mots, et quelles elles sont. Dans nos données, la répartition des structures semble assez similaire d'un enfant à l'autre.

Les comparaisons dans l'exemple de Marie (exemple 5), semblent montrer que cet enfant privilégie, dans l'ensemble, les structures VCV $(\mathrm{CV})$ pour les proto-mots et les mots malgré des proportions régulièrement plus importantes de structures $\mathrm{CVCV}(\mathrm{CV})$, pour les mots et les proto-mots. Mais il est notable que la proportion de VCV est bien plus importante pour les proto-mots que pour les mots. L'âge induit une différence de structure syllabique des productions. La différence perçue au niveau des proto-mots semble indiquer que la différence, avec l'âge, se situe au niveau d'une valorisation importante d'une structure VCV(CV) alors que nous avons affaire à l'inverse pour les mots. 


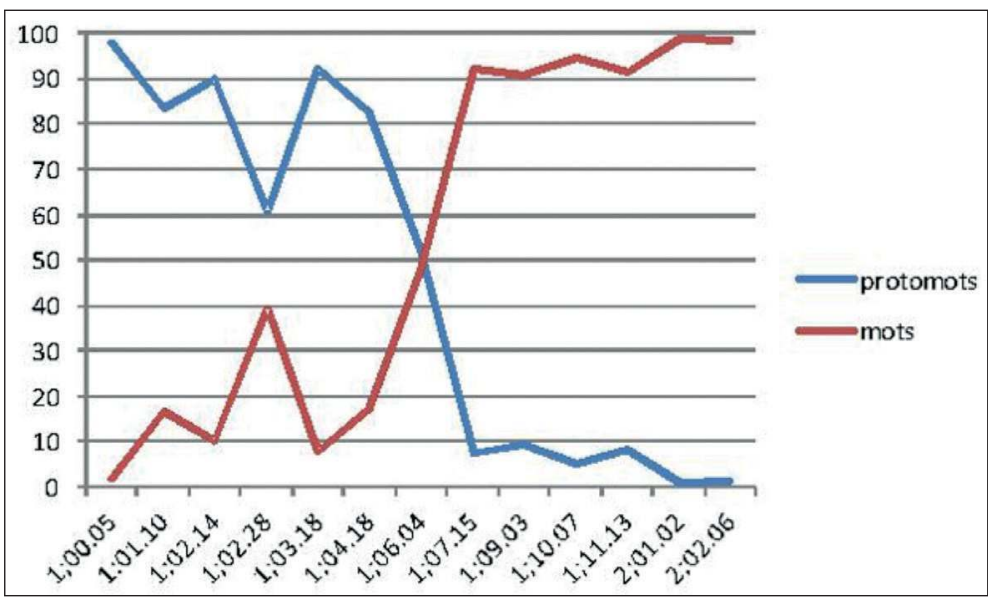

Exemple 5. - Comparaison des répartitions des structures simples VCV et CVCV des proto-mots et des mots de Marie en pourcentages de 1;00.03 à 2;00.09.

\subsection{L'acquisition des structures syllabiques complexes dans le passage des proto-mots aux mots}

Dans les structures complexes, concernant l'évolution des structures avec attaque branchante et syllabes avec coda, nous observons une augmentation régulière des syllabes avec coda tandis que les attaques branchantes semblent relever de la particularité du corpus (exemple 4). Nous y distinguons une préférence de Madeleine pour les codas plutôt que pour les structures branchantes, très peu représentées, et bien moins que chez Marie. Comme pour Marie, nous pouvons considérer l'augmentation significative des codas lors du moment de substitution des proto-mots aux mots, à 1;06.04. Ceci semble confirmer le fait que la substitution proto-mots/mots serait liée à une restitution plus régulière de schèmes syllabiques complexes. Pour Théophile, nous considérons les mêmes observations que Marie et Madeleine au niveau des codas et des branchantes, excepté le fait que Théophile semble présenter un profil différent. Théophile, contrairement à Marie et Madeleine, semble favoriser les branchantes plutôt que les codas au niveau des structures complexes. Mais il est également possible d'observer que l'augmentation des structures complexes est relative au moment de substitution des proto-mots par les mots. Au niveau de la composition des structures complexes, nous pouvons observer un profil encore différent des autres enfants dans la mesure où Elouan ne semble pas employer une structure 
plutôt qu'une autre. La répartition entre les codas et les branchantes semble assez proche et régulière. Comme pour les trois autres enfants, nous pouvons considérer une proportion plus importante après le phénomène de substitution proto-mots/mots si nous ramenons les résultats à l'ensemble des productions (exemple 6).

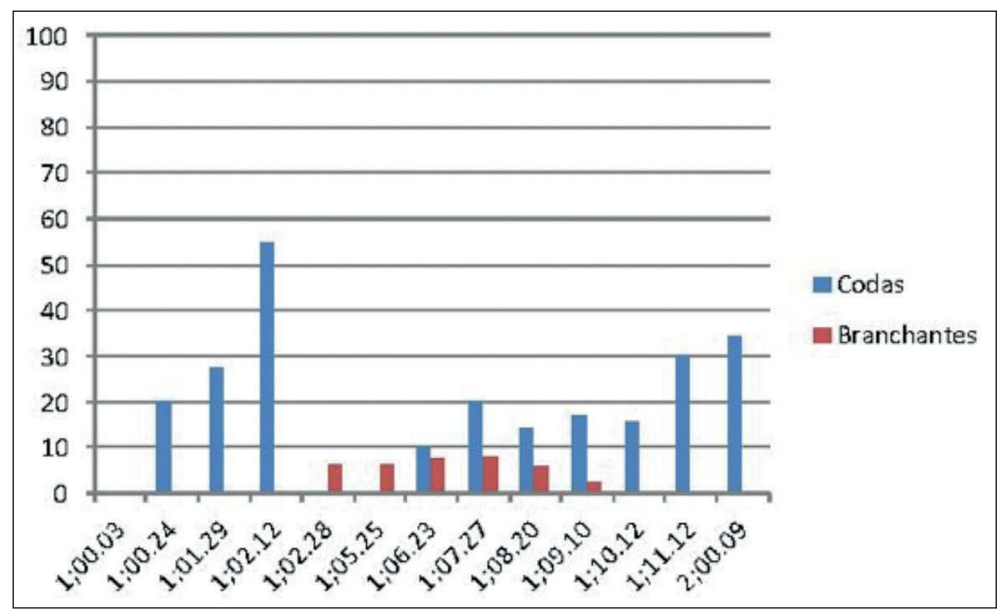

Exemple 6. - Évolution de la proportion de codas et de branchantes dans les productions de mots de Marie de 1;00.03 à 2;00.09.

\subsection{L'acquisition des structures articulatoires complexes dans la transition des proto-mots aux mots}

Au niveau des phonèmes, nous avons pu constater que, dans nos données, un certain nombre d'unités n'apparaissent jamais dans les productions de proto-mots : /R/, / / / /3/, toutes les voyelles nasales, et nous notons le /y/, /s/ et le /f/, qui apparaissent mais en proportion moindre (Wauquier-Gravelines, 2005 ; Recasens, 2013 ; dos Santos, 2007; Vihman, DePaolis \& Keren-Portnoy, 2009). Là aussi nous tenons à souligner qu'il ne peut s'agir d'un indice de généralité. Cependant, les données semblent bien indiquer une faible tendance de ces unités à apparaitre dans les proto-mots. Nous faisons ici le parallèle avec l'ordre d'apparition des sons; en effet, le /y/ et les voyelles nasales font partie de celles qui apparaissent le plus tard ou qui s'acquièrent sur la période la plus longue. Concernant les consonnes, les fricatives et les liquides apparaissent après les occlusives (Lambert, Rondal \& Sohier, 1980), ce que nous pouvons mettre en lien avec le fait que les $/ \mathrm{R} /, / \mathrm{J} /, / 3 /$, /s/ et /f/ sont en proportion moindre dans nos résultats. 
Si nous observons l'évolution de la restitution de voyelles dans les mots et les proto-mots d'Elouan, nous observons l'apparition d'un écart concordant avec le début du phénomène de substitution, comme chez les autres enfants (exemple 7).

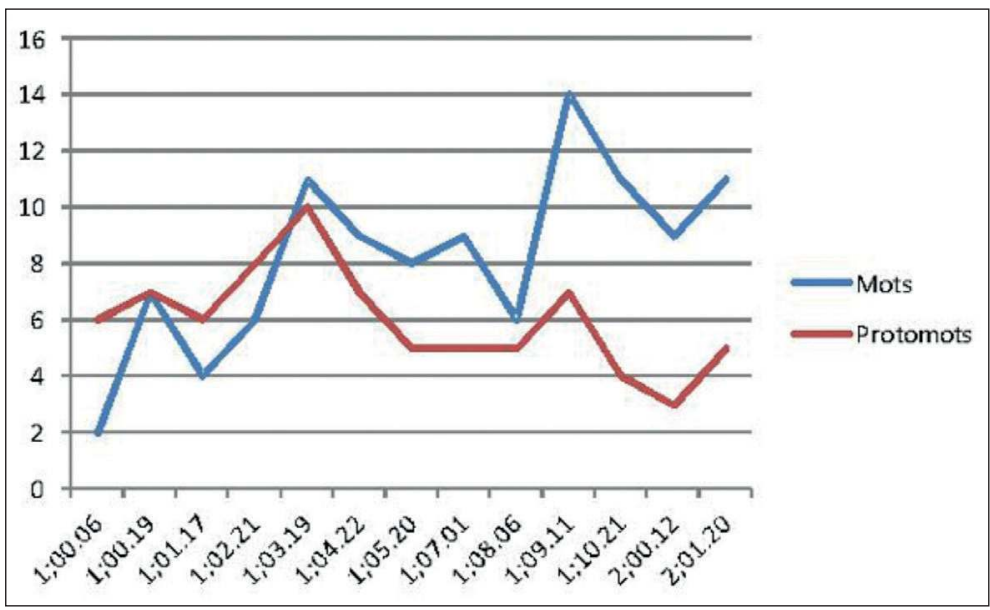

Exemple 7. - Évolution du nombre de voyelles différentes restituées dans les productions de mots et de proto-mots d'Elouan de 1;00.06 à 2;01.20.

L'augmentation du nombre de voyelles différentes maitrisées par l'enfant est à l'avantage des mots. Le nombre de voyelles différentes semble diminuer dans les productions de proto-mots. Le rapport mesurant la variabilité vocalique montre que l'écart s'accentue au profit des proto-mots. La restitution de mots augmentant, le nombre de voyelles augmentant, les productions de mots sont caractérisées par une stabilisation grandissante tandis que les proto-mots sont caractérisés par une variabilité grandissante. Notre étude s'étant basée sur des critères de production et de perception pour distinguer les mots des proto-mots, il peut sembler logique que l'adulte «naïf» ne caractérise comme mot que ce qui possède les propriétés les plus stables dans l'articulation de l'enfant, et donc que cette stabilisation soit à la faveur d'une maturation physiologique et phonologique (MacNeilage \& Davis, 1990; 2000; cf. partie 4.2).

Les voyelles privilégiées dans les mots sont généralement, pour les quatre enfants, /a/, /e/, /o/, / / /, / / /, /i/, /y/. Toutes les voyelles nasales sont présentes. La voyelle nasale ouverte /ã/ est présente. La voyelle 
la moins présente est le /œ/. Pour les proto-mots, nous trouvons, de façon privilégiée, /a/, /e/, /o/, /ə/, /i/. Les voyelles nasales sont toutes absentes. Les orales les moins répandues sont toutes les mi-ouvertes. Globalement, nous avons trouvé les mêmes similitudes chez les quatre enfants (exemple 8).

\begin{tabular}{|l|l|l|l|l|l|}
\hline Voyelles & $\mathrm{a}$ & è & $\mathrm{e}$ & $\mathrm{o}$ & $\mathrm{O}$ \\
\hline Mots & 40,0428266 & 6,42398287 & 14,7751606 & 5,1391863 & 1,49892934 \\
\hline Proto-mots & 47,4226804 & 5,44918999 & 13,8438881 & 12,371134 & 1,17820324 \\
\hline
\end{tabular}

\begin{tabular}{|l|l|l|l|l|l|}
\hline Voyelles & E & F & y & u & e \\
\hline Mots & 3,64025696 & 1,28479657 & 0,85653105 & 4,06852248 & 7,28051392 \\
\hline Proto-mots & 3,82916053 & 1,76730486 & 1,47275405 & 4,71281296 & 5,15463918 \\
\hline
\end{tabular}

\begin{tabular}{|l|l|l|l|l|l|}
\hline Voyelles & $\mathrm{i}$ & $\tilde{\mathrm{a}}$ & $\tilde{\mathrm{o}}$ & $\mathrm{C}$ & $\mathrm{D}$ \\
\hline Mots & 8,56531049 & 5,35331906 & 0,64239829 & 0,21413276 & 0,21413276 \\
\hline Proto-mots & 2,7982327 & & & & \\
\hline
\end{tabular}

Exemple 8. - Pourcentage des voyelles des proto-mots et des mots les quatre enfants confondus au stade de MLU I.

Généralement, les consonnes privilégiées dans les mots sont $/ 1 /, / \mathrm{m} /$, $/ \mathrm{n} /, / \mathrm{k} /, / \mathrm{p} /, / \mathrm{d} /, / \mathrm{t} /$. Les /3/, /R/, / / /, /f/, /v/ /s/ et /z/ sont en proportion importante (exemple 9).

\begin{tabular}{|l|l|l|l|l|l|l|l|}
\hline & p & b & t & d & k & g & f \\
\hline Mots & 4,11764706 & 7,94117647 & 11,4705882 & 5,88235294 & 6,47058824 & 7,35294118 & 0 \\
\hline Proto-mots & 1,98412698 & 7,53968254 & 19,2460317 & 20,2380952 & 4,76190476 & 5,15873016 & 0 \\
\hline
\end{tabular}

\begin{tabular}{|l|l|l|l|l|l|l|}
\hline & $\mathrm{v}$ & $\mathrm{s}$ & $\mathrm{z}$ & $\mathrm{S}$ & $\mathrm{Z}$ & $\mathrm{m}$ \\
\hline Mots & 1,76470588 & 7,05882353 & 2,64705882 & 2,94117647 & 0,29411765 & 22,9411765 \\
\hline Proto-mots & 5,35714286 & 0,79365079 & 0 & 0 & 0,5952381 & 3,76984127 \\
\hline
\end{tabular}

\begin{tabular}{|l|l|l|l|l|l|l|l|}
\hline & $\mathrm{n}$ & $\mathrm{l}$ & $\mathrm{R}$ & $\mathrm{J}$ & $\mathrm{j}$ & $\mathrm{w}$ & $\mathrm{H}$ \\
\hline Mots & 1,76470588 & 10,5882353 & 2,35294118 & 1,17647059 & 1,47058824 & 1,76470588 & 0 \\
\hline Proto-mots & 3,37301587 & 16,468254 & 0 & 0 & 6,15079365 & 4,56349206 & 0 \\
\hline
\end{tabular}

Exemple 9. - Pourcentage des consonnes des proto-mots et des mots les quatre enfants confondus au stade de MLU I. 
Dans les proto-mots, les enfants privilégient le $/ \mathrm{m} /, / \mathrm{t} /, / \mathrm{d} /, / \mathrm{k} /, / \mathrm{l} /, / \mathrm{b} /$ et $/ \mathrm{v} /$. Nous n'avons pas observé de $/ 3 /, / \mathrm{R} /, / \mathrm{J} /$ dans une seule transcription $^{5}$ et très peu de /s/ et /f/. Sur le plan consonantique, nous observons le même phénomène pour les quatre enfants, une inversion à l'avantage des mots, concordant avec le phénomène de substitution (exemple 10).

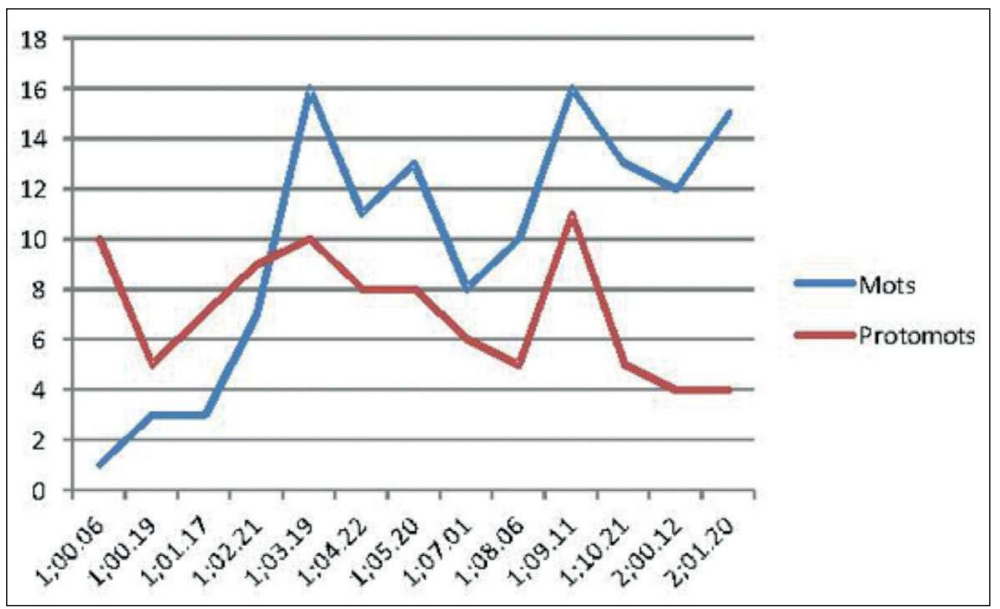

Exemple 10. - Évolution du nombre de consonnes différentes dans les productions de mots et de proto-mots d'Elouan de 1;00.06 à 2;01.20.

La mesure de la variabilité consonantique nous montre la même dynamique chez les quatre enfants. Le ratio est à l'avantage des protomots. Ainsi, plus l'enfant maitrise de consonnes, plus les productions de mots affichent une stabilité phonologique, et plus les proto-mots affichent de variabilité phonologique.

\section{Développement des premiers mots, théorie de l'optimalité et théorie du Cadre /Contenu}

\subsection{Le développement phonologique chez l'enfant}

Chez l'enfant, l'acquisition des éléments phonologiques commence avant le babillage, peut-être entre 4 et 6 mois comme le montrent les

5. Nous rappelons que ces éléments ne sont relatifs qu'à notre échantillon et non sur l'étendue de la transcription. 
études de perception, d'identification et de catégorisation basées sur les contrastes de sons entre les langues ou sur les enfants bilingues (Nazzi, 2006; Bosch, Ramon-Casas \& Gallés, 2006). Mais en production, ils commencent à être restitués en se conformant de plus en plus aux éléments de la langue adulte avec le babillage avancé et varié entre 10-12 mois (formes alternant une consonne et/ou une voyelle, comme [kapo]). Les travaux de Stoel-Gammon (1989) à partir de ceux de Oller et Eilers (1988) ont permis d'établir le calcul de niveau de babillage moyen (Mean Babbling Level), souvent appliqués pour déterminer des retards de langage (Morris, 2010). Par ailleurs, de nombreux travaux ont permis de recenser la présence et la fréquence d'un certain nombre de sons durant la période du babillage varié, surtout au niveau des consonnes (Davis \& MacNeilage, 1995; Boysson-Bardies \& Vihman, 1991). Ce sont les formes de ce babillage varié qui donneraient les premiers patrons de mots (Oller, 2000; Owens, 2008; Davis \& MacNeilage, 1995). Le babillage varié se prolonge et se stabilise en même temps que les premiers mots, entre 12-16 mois. À ce moment-là, des règles phonologiques plus complexes commencent à être acquises. Enfin, de 16 à 24 mois, le babillage varié tend à disparaitre et l'acquisition de la phonologie de la langue adulte passe au premier plan jusqu'à ce qu'elle soit supplantée par l'explosion lexicale. Concernant ces aspects et cette période, les travaux de MacNeilage (1998) se sont centrés sur les liens pouvant exister entre le babillage varié précédant la période des premiers mots, le babillage varié contemporain de la période des premiers mots et les premiers mots au niveau des productions combinant une consonne et une voyelle, en fonction du point d'articulation des consonnes (coronales, labiales, dorsales) et du lieu d'articulation des voyelles (avant, centre, arrière) (MacNeilage \& Davis, 1990; 2000; Davis \& MacNeilage, 1995). Ces travaux ont permis d'alimenter la Frame/Content Theory (théorie Cadre ou ossature/Contenu) à partir d'une continuité au niveau articulatoire et d'une prédictibilité des combinaisons articulatoires en fonction de la maturation de celles-ci. Plus récemment, nous pouvons citer les travaux de Kern et Davis (2009) et Davis et Kern (2011), prolongeant les travaux précédemment cités au niveau de la comparaison translinguistique (français, roumain, tunisien, turc et allemand). 


\subsection{Le développement phonologique, la théorie de l'optimalité et la théorie du Cadre/Contenu}

Dans notre travail, nous avons pu mettre en avant le fait que l'inversion des proportions des mots et des proto-mots et le fait que les mots deviennent les productions privilégiées en termes de proportions, sont corrélatifs de l'acquisition de règles phonologiques et de la restitution de structures syllabiques, consonantiques et vocaliques complexes. Afin de mieux observer l'évolution des proportions de proto-mots et de mots, nous avons fait le choix d'un corpus longitudinal permettant une observation régulière sur la durée. Or, notre corpus longitudinal a pu mettre en avant la rapidité avec laquelle se produit l'inversion entre les deux catégories de productions qui nous intéressent. De fait, pour expliquer cette vitesse de transition et d'inversion, nous avons eu recours, sur le plan syllabique, à l'OT et sur le plan articulatoire, à la théorie du Cadre/ Contenu.

Les règles phonologiques (Prince \& Smolenski, 1993; Tranel, 2000) concernent l'explication du fonctionnement d'une langue. Comment interpréter nos résultats en termes de règles ou de développement articulatoire? Quel positionnement théorique en permet le meilleur éclairage selon nous? Se peut-il qu'une complémentarité des deux approches soit possible ? Le choix de l'OT, de la théorie du Cadre/Contenu et de l'approche longitudinale, nous a conduit à envisager les raisons de l'inversion rapide des proportions de proto-mots et de mots au niveau phonologique et articulatoire. La réflexion épistémologique nous a permis ainsi de cerner, lorsque notre problématique a été soulevée, à la fois la pertinence du choix théorique et du choix méthodologique. La définition des proto-mots et des mots nous a conduit vers une approche longitudinale pour mieux révéler l'évolution de la coexistence de ces deux types de productions.

L'ensemble des classifications des types syllabiques selon les langues a été effectué dans le cadre de l'OT (Prince \& Smolensky, 2004). En français, le maintien ou la violation de règles est cohérent avec l'existence de certaines particularités, hiatus, assimilation, chute consonantique ou vocalique (Tranel, 2000). Le respect des règles peut ainsi conduire à certains phénomènes, comme la chute du [R] du mot livre dans la séquence «un livre tout jauni» (ibid., p. 48). De même, la prononciation de la coda est conforme à la règle générale, et sa nonprononciation considérée comme une transgression, comme dans le mot exact, que certains prononcent sans coda. Cette transgression possible rend ainsi la coda non pas obligatoire mais seulement facultative. 
La contrainte infléchit dans le sens de l'absence de coda. Ceci est rendu possible par le fait que les adeptes de l'OT ont tendance à effectuer un découpage en mores ${ }^{6}$ pour toutes les langues afin de mesurer la quantité syllabique, ou son poids (léger/lourd) en fonction du nombre de mores qui constitue le noyau ou la rime (McCarthy, 1979; Selkirk, 1982; Clements \& Keyser, 1983; Hayes, 1989, 1995).

Ces approches phonologiques sont aujourd'hui de première importance. Des travaux ont montré l'importance, dans la période qui précède celle des premiers mots, du babillage varié (Ferguson \& Farwell, 1975 ; McCune \& Vihman, 1987; Vihman, DePaolis \& Keren-Portnoy, 2009; Menn \& Stoel-Gammon, 2005; Oller \& Eilers, 1988) dans la transition vers les premiers mots. En effet, ce babillage varié fournit certains patrons syllabiques VCV et CVCV (Boysson-Bardies, 1993) qui seront ceux des premiers mots. Selon McCune et Vihman (1987), il n'est pas particulièrement question de contraintes dans le cadre de l'OT, mais plutôt de l'existence de filtres articulatoires permettant une restitution avant tout des schémas les plus habituels et les moins contraignants apparus pendant le babillage, appelés Vocal Motor Schemes. Ces schémas sont alors les plus proches de ceux de la langue adulte et servent à faciliter (bootstrap) l'acquisition des unités phonologiques. C'est dans ces structures déjà présentes dans le babillage que vont apparaitre les premiers mots (Vihman \& McCune, 1994). Cependant, nous notons que les caractéristiques du Syllable Structure Level (Kiparski, 1985; Ito, 1986; Borowski, 1989) ne sont appliquées que très rarement, et le plus souvent dans le cadre clinique des enfants à retard de développement ou des enfants sourds ayant reçu très tôt un implant cochléaire (Morris, 2010).

Dans les premières productions linguistiques de l'enfant, cet enchainement syllabique correspond, au niveau phonologique, au mot

6. La more est une unité phonologique de niveau inférieur à la syllabe qui sert à déterminer le poids de la syllabe en fonction du nombre de mores que représente le noyau de cette syllabe (le noyau peut, selon les langues, être une consonne ou une voyelle; dans ce cas, une voyelle ou une consonne brève représente une more et une voyelle ou une consonne longue représente deux mores); selon les langues, la coda peut représenter une more, comme en japonais (la coda est un élément facultatif qui suit le noyau de la syllabe et qui peut être une voyelle ou une consonne selon le système phonologique de la langue; en français, la coda est constituée uniquement de consonnes; la coda est l'élément qui définit les syllabes fermées). 
phonologique (Demuth, 1995; Pater, 1997). La structure de ce mot phonologique semble préservée des transgressions de contraintes par les enfants français (Demuth \& Johnson, 2003; dos Santos, 2007). Ceci conduit même les enfants à des réductions syllabiques, passant des dissyllabes aux monosyllabes (bien que le mot cible soit dissyllabique) : lorsque les contraintes ne sont pas encore maitrisées ou trop difficiles à transgresser, l'enfant a recours à une production plus simple au niveau phonologique et articulatoire, mais ayant cependant des caractéristiques communes avec l'élément cible. Par ailleurs, il semblerait que ce mot phonologique, dans de nombreuses langues, même en français, réponde à une structure en pieds (Pater, 1997).

Nous avons ainsi observé des tendances qui semblent rassembler les quatre enfants. Ces dernières tendances conduisent à mettre en avant une préférence à la simplification pour les proto-mots, dénués de toute structure syllabique complexe, et avec une proportion de structures $\mathrm{VCV}(\mathrm{CV})$ en augmentation avec le temps. Ce sont des productions qui ont tendance à répondre à la simplification phonologique (Menn \& Stoel-Gammon, 2005), à des constructions régulières et correspondant aux structures de base qui n'enfreignent aucune règle dans le cadre d'une approche par l'OT. De plus, la persistance de proto-mots dans la période où les mots sont les productions majoritaires, nous montre qu'ils sont toujours systématiquement réalisés via ces formes simples ne violant aucune règle. Par ailleurs, il pourrait exister une progression dans l'acquisition des transgressions : dans le cadre du Syllable Structure Level (Kiparski, 1985; Ito, 1986; Borowski, 1989), nous pourrions alors considérer que les proto-mots restent au niveau 1 et que l'acquisition des mots, lors du phénomène de substitution, implique l'acquisition de structures de niveau 2. Le niveau définit la structure de la syllabe; dans le niveau 1 elle est composée d'une voyelle voisée, d'une consonne syllabique voisée ou d'une syllabe CV dans laquelle la consonne est une glottale ou une glide; le niveau 2 est constitué de syllabes de type CV, VC ou CVC avec un seul type de consonne; le niveau 3 est constitué de syllabes avec deux types de consonnes différents ou plus (Olswang, Stoel-Gammon, Coggins \& Carpenter, 1987).

\subsection{Variabilité et stabilité phonologique dans l'acquisition des premiers mots}

Notre objectif a été d'observer ce qui distingue ou rapproche les protomots des mots sur le plan phonologique. Il a été difficile d'établir des 
phénomènes de simplification phonologique (Menn \& Stoel-Gammon, 2005) ou de réduction syllabique dans les proto-mots dans la mesure où ce qui les caractérise, selon notre définition, c'est la variabilité des phonèmes qui constituent les structures syllabiques et l'impossibilité d'y identifier une quelconque morphologie. Mais il nous apparait intéressant de comparer les régularités phonologiques de chaque catégorie, proto-mots et mots et d'essayer d'observer ce qui peut justifier le phénomène de substitution que nous avons mis en évidence.

Dans leur acquisition, les mots sont caractérisés par une augmentation du nombre de voyelles et de consonnes maitrisées par les quatre enfants, tandis que les proto-mots sont caractérisés par une variabilité vocalique et consonantique grandissante selon l'évolution du ratio observé. Inversement, bien que restituant de plus en plus de voyelles et de consonnes, les mots se caractérisent par l'augmentation de la stabilité phonologique de ce type de productions. Nous pouvons alors observer de façon assez explicite où se situent les éléments impliqués dans le phénomène de substitution proto-mots/mots.

\subsection{Le développement articulatoire et phonologique}

L'hypothèse permettant, en premier lieu, d'expliquer ce phénomène est alors relative au développement des capacités articulatoires de l'enfant et du lien entre le nombre de phonèmes assimilés et le nombre de mots restitués. Dès lors, la relation entre l'acquisition du lexique et du système phonologique (et des capacités articulatoires) semble s'établir (Kern \& Davis, 2009; Davis \& Kern, 2011; MacNeilage \& Davis, 1990, 2000; McCune \& Vihman, 1987, 2001). Si nous considérons également les critères du Mean Babbling Level, sans avoir à établir les calculs, nous pouvons considérer l'existence de deux niveaux différents entre les proto-mots et les mots par l'existence de consonnes et de voyelles dans les mots qui ne sont pas présentes dans les proto-mots.

\subsection{Les structures simples de mots}

Concernant les structures simples des mots, il y a aussi des différences importantes selon les proportions des différentes structures syllabiques avec le temps. De fait, l'OT a permis d'appuyer, ici, deux points : la caractérisation du passage aux mots par une complexification du système syllabique, et le fait que le recours aux proto-mots repose sur la restitution d'un pattern syllabique simple de base. Cette considération s'inscrit dans une relation également articulatoire, via les Vocal Motor 
Schemes (McCune \& Vihman, 1987), impliquant, dans le passage aux mots, une relation entre articulation et abstraction phonologique par la maitrise du contrôle moteur de certaines régularités articulatoires.

De fait, nous nous sommes appuyé sur les travaux de McCune et Vihman $(1987,2001)$ et de MacNeilage et Davis (1990) au niveau des VMS et de la théorie du Cadre/Contenu relativement au développement articulatoire. En effet, selon nous les considérations ne peuvent pas concerner le plan purement abstrait (phonologique). Le développement observé sur l'exemple 1 permet de relier l'aspect phonologique à l'aspect articulatoire. Au niveau phonologique, nous avons relevé une absence totale de phonèmes de type $/ \mathrm{R} /, / \mathrm{J} /, / 3 /$ et de voyelles nasales dans les proto-mots (y compris dans ceux de la période où les mots sont les productions majoritaires) alors qu'ils sont présents dans les mots. La plus grande complexité des sons restitués par l'enfant dans les mots que dans les proto-mots permet d'envisager la maitrise plus grande de régularités articulatoires et d'en prédire le développement dans les mots. Cependant, notons que les proto-mots, se réalisant sur des bases articulatoires plus simples, rappellent les aspects de l'OT, bases de notre analyse au niveau syllabique.

Cette tendance à la simplification est notamment appuyée par la présence persistante de proto-mots dans la période où les mots sont les productions largement majoritaires. Toutefois, la stabilisation des éléments phonologiques et articulatoires est corrélée, dans nos données, à l'explosion lexicale; autrement dit, nous avons deux possibilités : soit l'explosion lexicale n'apparait que lorsque les phénomènes de maturation phonologique et articulatoire ont franchi une étape dans le courant de la deuxième année de l'enfant, soit les phénomènes phonologiques articulatoires franchissent une étape sous l'influence de l'explosion lexicale.

\section{Conclusion}

Notre travail a porté sur l'observation et la comparaison des productions porteuses de sens qui coexistent dans la période des premiers mots, les proto-mots et les mots. Notre approche n'a pas consisté en un positionnement théorique immédiat, mais elle a déterminé la définition de chaque catégorie par rapport aux travaux précédents, depuis ceux de Menn (1978). En nous basant sur les travaux de Vihman et McCune (1994), notamment sur leurs critères méthodologiques qui permettent d'accepter ou de rejeter un candidat-mot comme mot et non 
un proto-mot, nous nous sommes retrouvé face à la nécessité de mieux établir ce à quoi correspondait un proto-mot par rapport à un mot. Notre première approche s'est donc basée sur un test de perception auprès de dix adultes afin de confirmer et dissocier l'existence de ces deux catégories. Une fois ce travail effectué, nous avons pris le parti d'utiliser un corpus longitudinal qui nous a permis d'observer l'évolution de ces deux catégories de productions auprès de 4 enfants français monolingues. L'observation de leur évolution dans le temps nous a permis de révéler l'existence d'une phase rapide de substitution durant laquelle les mots, en termes de proportions, se substituent aux proto-mots, sans transition graduelle ou progressive particulière chez nos 4 enfants. Dans la mesure où le test de perception a révélé qu'un des éléments distinctifs majeurs entre ces deux catégories se situait au niveau de la forme sonore, c'est alors que, pour expliquer ce phénomène rapide révélé par notre corpus longitudinal, nous avons eu recours, pour comparer ces deux catégories, à l'OT concernant la dimension syllabique, et à la théorie du Cadre/Contenu concernant la dimension articulatoire.

Ainsi, dans notre étude, la question principale est de savoir ce qui justifie le renversement des proportions de proto-mots et de mots. Selon les définitions données par Vihman et McCune (1994) que nous avons retenues et du test de perception que nous avons réalisé, la distinction proto-mot/mot se situe au niveau de la forme sonore (dimension articulatoire) et syllabique (dimension phonologique). Ainsi, les différences peuvent-elles impliquer une complexification syllabique concernant les mots, par l'acquisition de structures complexes qui ne sont pas celles de base dans le babillage et les proto-mots? Notre questionnement nous a conduit à envisager divers cadres théoriques, comme l'OT (Prince \& Smolenski, [1993] 2004), les théories basées sur l'usage appliquées à la phonologie (Bybee, 2002; Chevrot, Fayol \& Laks, 2005), les approches morphologiques (Wauquier-Gravelines, 2002) ou le développement phonologique dynamique (Vihman, DePaolis \& Keren-Portnoy, 2009). Notre choix théorique s'est alors porté sur l'OT que nous avons jugée mieux adaptée à l'explication du phénomène que nous avons mis en évidence : il nous fallait justifier comment, dans le passage rapide des proto-mots aux mots (fig. 1), on passe aussi vite de proto-mots ne possédant pas de structures syllabiques complexes à des mots possédant des structures syllabiques complexes : absence totale de syllabes avec attaques branchantes ou codas dans les proto-mots alors que ces types de syllabes sont présentes dans les mots.

L'utilisation conjointe de deux cadres théoriques différents pour expliquer le développement syllabique et articulatoire s'est imposé par 
les résultats obtenus. Nous pouvons poser la question de la complémentarité de ces deux cadres théoriques. Dans des travaux antérieurs (Roux, 2012, 2014), nous avions montré que si l'on fait abstraction de l'inversion des courbes dont nous parlions précédemment et de la distinction proto-mots /mots, l'enfant produit autant de productions porteuses de sens avant et après le renversement des courbes. Par ailleurs, les productions porteuses de sens sont antérieures aux premiers mots (Veneziano, 1981). Nous pensons que le découpage en étapes «premiers mots» et «explosion lexicale» est peut-être trop fort dans la mesure où nous pouvons observer la présence de multiples productions porteuses de sens avant les premiers mots et que leur proportion est identique avant et après l'explosion lexicale. De fait, les évolutions seraient plus spécifiquement liées aux évolutions phonologiques et articulatoires dans la mesure où la dimension sémiotique est déjà présente. Les résultats de la présente étude nous ont conduit à envisager deux approches, l'OT pour la dimension syllabique et la théorie du Cadre/Contenu pour la dimension articulatoire. L'opposition entre les proto-mots et les mots repose principalement sur ces caractéristiques. La présence de règles (et de contraintes), au niveau des structures syllabiques et d'une maturation articulatoire au niveau des sons produits, ne sont pas incompatibles bien que relevant de deux cadres théoriques différents. Le positionnement de McCune et Vihman (1987) permet la mise en évidence des schémas vocaux les plus simples (VMS) dès le babillage et restitués dans les premiers mots, et le niveau de structure syllabique (Kiparski, 1985; Ito, 1986) met en avant l'existence de niveaux de structures syllabiques dont nous pouvons dire que ceux-ci sont contraints selon le système phonologique de la langue. Ces approches, au regard de nos résultats, rendent possible la perspective d'une complémentarité des deux cadres théoriques qui se sont imposés à nous en fonction de nos résultats.

Les problèmes rencontrés ont plus été inhérents à l'identification des catégories de productions, nécessitant de recourir à un test de perception. Par ailleurs, l'analyse longitudinale s'est avérée à même de mettre en avant un phénomène de substitution auquel nous avons répondu en mobilisant la théorie de l'OT et la théorie du Cadre/Contenu. L'échantillonnage de notre corpus longitudinal est limité sur le plan quantitatif dans la mesure où, pour des raisons de temps et de travail, nous nous sommes limité à 4 enfants. En connaissance des données révélées, cette étude mériterait d'être développée dans une perspective quantitative via des relevés transversaux à trois moments différents, et avec des enfants de même niveau de MLU pour chaque relevé. Nous pourrions 
ainsi affiner le phénomène de substitution que nous avons observé, et pousser plus le détail sur le plan syllabique et articulatoire dans l'OT et la théorie du Cadre/Contenu.

\section{RÉFÉRENCES BIBLIOGRAPHIQUES}

Arborio, Anne-Marie \& Fournier, Pierre. (1999). L'enquête et ses méthodes : l'observation directe. Paris : Nathan Université.

Bloom, Lois. (1973). One Word at a Time: The Use of Single Word Utterances before Syntax. La Haye, Pays-Bas : Mouton.

Borowski, Toni. (1989). Structure Preservation and the Syllable Coda in English. Natural Language and Theory, 7, 145-166.

Bosch, Laura, Ramon-Casas, Marta \& Sebastián Gallés, Nuria. (2006). Catégories phonologiques et représentation des mots dans le développement lexical de l'enfant bilingue. Recherches linguistiques de Vincennes, 35, 53-76.

Boysson-BARDiEs, Bénédicte (de). (1993). Ontogeny of Language-Specific Syllabic Production. Dans B. de Boysson-Bardies, S. de Schoen, P. Jusczyk, P. F. MacNeilage \& J. Morton (dir.), Developmental Neurocognition: Speech and Face Processing in the First Year of Life (p. 353-363). Dordrecht : Kluwer Academic Publishers.

Boysson-Bardies, Bénédicte (de) \& Vihman, Marilyn May. (1991). Adaptation to Language: Evidence from Babbling and First Words in Four Languages. Language, 67, 297-319.

BRown, Roger. (1973). A First Language: The Early Stages. Cambridge : Harvard University Press.

B ybee, Joan. (2002). Phonological Evidence for Exemplar Storage of Multiword Sequences. Studies in Second Language Acquisition, 24(2), 215221.

Chevrot, Jean-Pierre, Fayol, Michel \& LaKs, Bernard. (2005). La liaison : de la phonologie à la cognition. Langages, 158, 3-7.

Сномsкy, Noam. ([2000] 2005). Nouveaux horizons dans l'étude du langage et de l'esprit. Paris : Stock.

Clements, G. Nick \& Keyser, Samuel Jay. (1983). CV Phonology. A Generative Theory of the Syllable. Cambridge : MIT Press.

Davis, Barbara L. \& Kern, Sophie. (2011). A Complexity Theory Account of Canonical Babbling. Dans McLeod \& Goldstein (dir.), Young Children, Multilingual Aspects of Speech Sound Disorders in Children. Multilingual Matters Publishers.

Davis, Barbara L. \& MacNeilage, Peter F. (1995). The Articulatory Basis of Babbling. Journal of Speech and Hearing Research, 38, 1199-1211. 
Demuth, Katheryn. (1995). Markedness and the Development of Prosodic Structure. Dans J. Beckman (dir.), Proceedings of the North East Linguistic Society (25, p. 13-25). Amherst, MA : GLSA, University of Massachusetts.

Demuth, Katheryn \& Johnson, Mark. (2003). Truncation to Subminimal Words in Early French. Canadian Journal of Linguistics, 48, 211-241.

DePaolis, Rory A. \& Keren-Portnoy, Tamar. (2009, mai). The Interaction of Production and Perception Skills in Infancy and Their Effects on Word Learning. Communication présentée au $17^{\mathrm{e}}$ Manchester Phonology Meeting, Manchester, Angleterre.

Ferguson, Charles A. \& Farwell, Carol B. (1975). Words and Sounds in Early Language Acquisition. Language, 51, 419-439.

Halliday, Michael Alexander Kirkwood. (1975). Learning How to Mean. Londres : Edward Arnold.

Hayes, Bruce. (1989). The Prosodic Hierarchy in Meter. Dans P. Kiparsky \& G. Youmans (dir.), Rythm and Meter (p. 201-260). Orlando : Academic Press.

Hayes, Bruce. (1995). Metrical Stress Theory: Principles and Case Studies. Chicago : University of Chicago Press.

Iто, Junko. (1986). Syllable Theory in Prosodic Phonology. New York : Garland Publishing.

Kern, Sophie \& Davis, Barbara L. (2009). Emergent Complexity in Early Vocal Acquisition: Cross-Linguistic Comparisons of Canonical Babbling. Dans F. Pellegrino, E. Marsico, I. Chitoran \& C. Coupé (dir.), Approaches to Phonological Complexity. Mouton de Gruyter.

Kern, Sophie \& Gayraud, Frédérique. (2007). Caractéristiques phonologiques des noms en fonction de l'âge d'acquisition. Enfance, 59, 324338.

KIPARSKY, Paul. (1985). Remarks on the Metrical Structure of the Syllable. Dans W. Dressler, O. Pfeiffer \& J. Rennison (dir.), Phonologica 1980: Proceedings of the Fourth International Conference on Phonology (p. 245-256).

LAmbert, Jean-Luc, Rondal, Jean-Adolphe \& Sohier, C. (1980). Analyses des troubles articulatoires chez les enfants arriérés mentaux. Bulletin d'Audiophonologie, 10, 11-20.

Le Normand, Marie-Thérèse. (1997). Early Morphological Development in French Children. Dans A. S. Olofsson \& S. Strömqvist (dir.), Learning Disorders As a Barrier to Human Development: Cross-Linguistic Studies of Dyslexia and Early Language Development (p. 59-79). Luxembourg : Office for Official Publication of the European Communities.

MacNeilage, Peter F. (1998). The Frame/Content Theory of Evolution of Speech Production. Behavioral and Brain Sciences, 21, 499-546. 
MacNeilage, Peter F. \& Davis, Barbara L. (1990). Acquisition of Speech Production: Achievement of Segmental Independence. Dans W. I. Hardcastle \& A. Marchal (dir.), Speech Production and Speech Modeling (p. 55-68). Dordrecht : Kluwer.

MacNeilage, Peter F. \& Davis, Barbara L. (2000). On the Origin of the Internal Structure of Words Forms. Science, 288, 527-531.

McCARThy, John. (1979). On Stress and Syllabification. LI, 10, 443-466.

McCune, Lorraine \& Vinman, Marilyn May. (1987). Vocal Motor Schemes. Papers and Reports in Child Language development, 26, 72-79.

McCune, Lorraine \& Vinman, Marilyn May. (2001). Early Phonetic and Lexical Development: A Productivity Approach. Journal of Speech, Language, and Hearing Research, 44(3), 670-684.

Menn, Lise. ([1976] 1978). Pattern, Control, and Contrast in Beginning Speech: A Case Study in the Development of Word Form and Word Function. University Mocrofilms international.

Menn, Lise \& Stoel-Gammon, Carol. (2005). Phonological Development: Learning Sounds and Sound Patterns. Dans J. Berko Gleason (dir.), The Development of Language (p. 62-111). Needham Heights, MA : Pearson.

Morgenstern, Aliyah \& Parisse, Christophe. (2012). The Paris Corpus. French Language Studies, 22(1), 7-12.

Morgenstern, Aliyah. (2009). L'enfant dans la langue. Paris : Presses de la Sorbonne Nouvelle.

Morris, Sherrill R. (2010). Clinical Applications of the Mean Babbling Level and Syllable Structure. Language, Speech, and hearing Services in School, 41, 223-230.

NAzzi, Thierry (2006). Spécificité phonétique : de la perception précoce à l'acquisition des premiers mots. Recherches linguistiques de Vincennes, $35,31-52$.

Oller, D. Kimbrough. (2000). The Emergence of the Speech Capacity. Mahwah, NJ : Lawrence Erlbaum Associates.

Oller, D. Kimbrough \& EILERs, Rebecca E. (1988). The Role of Audition in Infant Babbling. Child Development, 59, 441-449.

Olswang, Lesley, Stoel-Gammon, Carol, Coggins, Truman \& Carpenter, Robt. (1987). Assessing Prelinguistic and Early Linguistic Behaviors in Developmentally Young Children. Seattle, WA : University of Washington Press.

Owens, Robert E., Jr (2008). Language Development: An Introduction. Londres : Pearson Educational International.

PAter, Joe. (1997). Minimal Violation and Phonological Development. Language Acquisition, 6(3), 201-253. 
Prince, Alan \& Smolensky, Paul. ([1993] 2004). Optimality Theory: Constraint Interaction in Generative Grammar. New York : Blackwell.

Recasens, Daniel. (2013). On the Articulatory Classification of (Alveolo) palatal Consonants. Journal of the International Phonetic Association, 43(1), 1-22.

Roux, Guillaume. (2012). Prélinguistique et linguistique dans la période des premiers mots : approches historique, épistémologique et expérimentale (Thèse de doctorat). Université Paul-Valéry, Montpellier.

Roux, Guillaume. (2014). Catégoriser les productions de la période des premiers mots de l'enfant. Faits de Langues, 43, 157-178.

Santos, Christophe dos. (2007). Développement phonologique en français langue maternelle (Thèse de doctorat non publiée). Université Lyon 2, Lyon.

SElkirk, Elizabeth. (1982). Syllables. Dans H. Hulst \& N. Smith (dir.), The Structure of Phonological Representations (Part II, p. 337-383). Dordrecht : Foris.

Stoel-Gammon, Carol. (1985). Phonetic Inventories 15-24 Months: A Longitudinal Study. Journal of Speech and Hearing Research, 28, 505-512.

Stoel-Gammon, Carol. (1989). Prespeech and Early Speech Development of Two Late Talkers. First Language, 9, 207-223.

Tomasello, M. (2003). Constructing a Language: A Usage-Based Theory of Language Acquisition. Cambridge : Harvard University Press.

Tranel, Bernard. (2000). Aspects de la phonologie du français et la théorie de l'optimalité. Langue française, 126, 39-72.

Veneziano, Eddy. (1981). Early Language and Nonverbal Development: A Reassessment. Journal of Child Language, 8, 541-563.

Vihman, Marilyn May, DePaolis, Rory A. \& Keren-Portnoy, Tamar. (2009). Babbling and Words: A Dynamic Systems Perspective on Phonological Development. Dans E. L. Bavin (dir)., The Cambridge Handbook of Child Language (p. 163-182). Cambridge : Cambridge University Press. Vinman, Marilyn May \& McCune, Lorraine. (1994). When Is a Word a Word? Journal of Child Language, 21(3), 517-542.

Wauguier-Gravelines, Sophie. (2002). Statistical Learning or Phonological Generalisation: The Case of Acquisition of Liaison in French. Communication présentée à la Special Session "Acquisition" of Tenth Phonology Meeting of Manchester (23 mai 2002).

Wauquier-Gravelines, Sophie. (2005). Acquisition et développement phonologiques. Dans N. Nguyen, S. Wauquier-Gravelines \& J. Durand (dir.), Phonologie et phonétique : forme et substance. Hermès. 